\title{
Me-Friends-Web (MFW): A Model for Navigation Assistance Through Social Navigation Networks
}

\author{
Hassan A. Karimi \\ Geoinformatics Laboratory, School of Information Sciences \\ University of Pittsburgh \\ 135 N. Bellefield, Pittsburgh, PA, 15260, US. \\ hkarimi@sis.pitt.edu
}

\begin{abstract}
Modern navigation systems/services are computationally sophisticated gadgets capable of assisting people with mobility in locations where digital road network maps are available. However, despite much advancements in navigation systems/services, they fall short of handling common constraints (e.g., individual preferences, indoor navigation, multimodal transportation, etc.) or the needs of people with specific limitations (e.g., wheelchair users, elderly, etc). Increasing the scope of navigation assistant goes beyond just adding more data or improving existing algorithms, rather new perspectives in requesting and receiving navigation assistance are needed. In this paper, we present a social networking perspective in navigation assistance in which members of the social network can share their navigation experiences. We discuss a navigation network system, called SoNavNet, and a model for it which integrates knowledge and experiences of the user (called "Me"), knowledge and experiences of friends through social networks (called "Friends"), and computation and information available through the Web (called "Web"). The model is called MeFriends-Web (MFW) and its goal is to overcome the shortcomings of modern navigation systems/services and facilitate personalized navigation assistance.
\end{abstract}

Keywords- Navigation Assistance, Navigation Experience, Personalized Navigation, Social Navigation Network

\section{INTRODUCTION}

$\mathrm{W}$ E define navigation as any activity requiring locations and the means to reach to them. Examples of navigation activities that fit this definition include points of interest (POIs), routes, and directions. Considering this definition, mobility is a term that we use interchangeably with navigation. People routinely require assistance for mobility when travelling in unfamiliar places, visiting a place for the first time, or travelling in complex places (these are places where users often are faced with difficulties in making navigation decision). For this, navigation assistance has become an integral aspect of daily life supported by in-car navigation systems/services and route planning has been possible through Web Mapping Services (WMSs), among other Web resources. Once considered an extravagant luxury, navigation systems/services nowadays play an important role in the decisions people make with respect to mobility and mobility planning. However, modern navigation systems/services remain primarily algorithmic with limited other means for formulating optimal navigation assistance. An ideal navigation system/service would allow for navigation operation both indoors and outdoors, support features to meet the navigation needs of individuals who are physically, cognitively, and sensory impaired, dynamically respond to changes in mode of transportation (e.g., from driving to walking to biking), and provide personalized navigation assistance.

Modern navigation systems/services, while commonplace, are not ideal as they lack key features and fall short of handling some of the capabilities listed above. In other words, there is a gap between the ideal navigation assistance and the navigation assistance currently supported through navigation systems/services. To overcome the shortcomings of modern navigation systems/services and making strides toward being able to provide the means for ideal navigation assistance, we have developed a social navigation network system, called SoNavNet [1]. SoNavNet, as an online social network system, facilitates communication among its members to share navigation experiences. To provide optimal personalized navigation assistance in SoNavNet, a model called MeFriends-Web (MFW) is developed. MFW provides navigation assistance that otherwise is not available and possible through existing navigation systems/services. With MFW, members of SoNavNet can be provided navigation assistance through coupling user's needs, interests, preferences, knowledge and experiences (thus "Me”), assistance that friends can provide (thus, "Friends"), and assistance that can be expected through online databases, WMSs (such as Google Maps, Yahoo Maps, Bing Maps), and sensor networks (thus "Web”). The premise of MFW is based on two observations: (a) algorithmic solutions that can provide the ideal navigation assistance may not be feasible due to several technical and non-technical issues and (b) social networking has already become widespread enabling the possibility of tackling some of the challenges that computation alone can not. For these reasons, MFW in SoNavNet considers all possible resources, i.e., what people know and what machines can compute, in formulating optimal navigation assistance solutions. MFW is a novel and viable approach in providing navigation assistance as it addresses the needs of navigation, simple to complex, by 
considering all possible resources including what the user needs, what and how much the user can be assisted by social networks, especially when the element of "trust" is considered, and what can be computed from map data. In this regard, MFW is a paradigm shift in navigation assistance addressing the needs of people at general, group, and individual levels. An example challenge where MFW in SoNavNet is well suited to address is a navigation application for those people with special requirements and/or unique preferences.

\section{MOTIVATION}

Some of the reasons for the gap between the ideal navigation assistance and the navigation assistance currently supported by navigation systems/services are as follows. Current navigation systems/services cannot address the specific needs of individuals including those with mobility, cognitive, and sensory impairments; cannot provide navigation assistance seamlessly in both indoors and outdoors; cannot adapt to different navigation requirements, such as navigation in daylight versus dark, navigation in rush hours versus off-peak hours, or navigation in snowy days versus sunny days; and cannot seamlessly adjust between different modes of transportation, such as driving, walking, bike riding, riding wheelchairs, or having limited mobility for stairs and other hindrances.

To realize how existing navigation systems/services fall short of handling certain navigation activities and how MFW can fill the gap and provide ideal navigation assistance in SoNavNet, a scenario on disability support is presented as follows. Mary, a manual wheelchair user, has moved into Pittsburgh to attend the University of Pittsburgh. Since it was her first time in Pittsburgh, she used her smartphone to join an online paraplegic community in Pittsburgh. In order to attend her first class, she inquired to find directions from her apartment to her classroom. MFW offered her directions that modified the shortest computed route to follow an evensurface sidewalk, taking her to the accessible entrance of the building as recommended by one of the trusted participants in the network. Upon entering the building, SoNavNet provided her with directions to the nearest elevator and suggested the proper hallway to arrive to her classroom. After the class, she decided to buy a textbook for class and asked SoNavNet to find her the nearest bookstore, which had the textbook in stock. SoNavNet located the bookstore and offered an accessible route to the bookstore. When she arrived at the store, she noticed that the bookstore had special offers on some textbooks, thus, she geotagged the bookstore, and later map marked and posted the special offer with a seven-day validity, so that other members of her social network could be alerted to and benefit of the same deal.

\section{BACKGROUND}

Existing navigation systems/services have evolved from specialized computer-based systems with specific basic navigation functions to sophisticated gadgets featuring a variety of options. While conventional navigation systems featured interfaces with limited functionality, were importable, and were only available for drivers, modern navigation systems/services offer flexible interfaces, are portable on handheld devices (including cell phones), and are available for pedestrians as well. Furthermore, through wireless networks, modern navigation systems/services are able to tap into real-time information such as traffic and road closures.

Despite much advancements in navigation systems/services, they are not universal in that they are only suitable for general navigation or specific populations. As a result, there have been developments of navigation systems/services, each to meet the requirements of a certain application or for the needs of certain population.

\section{SOCIAL NAVIGATION NETWORKS}

Future navigation systems/services should be universal [2] and [3]. They should meet the needs of all users including those with specific limitations, be adaptable to users with different computing knowledge, provide navigation assistance seamlessly in both indoors and outdoors, support navigation assistance suitable for various situations, provide individuals with proper assistance seamlessly based on the context, be capable of adjusting to different modes of transportation (such as driving cars, walking, riding bicycles, riding wheelchairs or Segways), and effectively be used in different countries with dissimilar policies and cultures. A new navigation assistance perspective that can support these requirements is social networking. We have developed a new social navigation network system in the Geoinformatics Laboratory of the School of Information Sciences at the University of Pittsburgh. This new system is called SoNavNet, short for social navigation network, whose main goal is to facilitate sharing of navigation experiences of its members. SoNavNet has the potential for supporting the capabilities and features of the ideal navigation assistance. SoNavNet allows members of the network to recommend and request navigation information including geotagged POIs, routes, directions, and geofences. For a detailed description of SoNavNet including architecture, components, and features refer to [1]. For an overview of the concept of SoNavNet and how it differs from existing navigation systems/services refer to [4]. For example applications of SoNavNet, collaborative mapping and education, refer to [5] and [6], respectively. For a description of the underlying model upon which SoNavNet is based refer to [7].

In this paper, we focus on MFW that allows navigation assistance through user's knowledge and experience, knowledge and experiences of user's friends, and information available through WMSs and other Web resources.

\section{MFW MODEL}

MFW addresses the universality aspect of navigation by taking a novel approach that combines user's knowledge with the assistance that is possible through friends and the information that is available through the Web (e.g., Google Maps and Bing Maps). MFW is focused on key activities of navigation that include POIs, routes, and directions. 


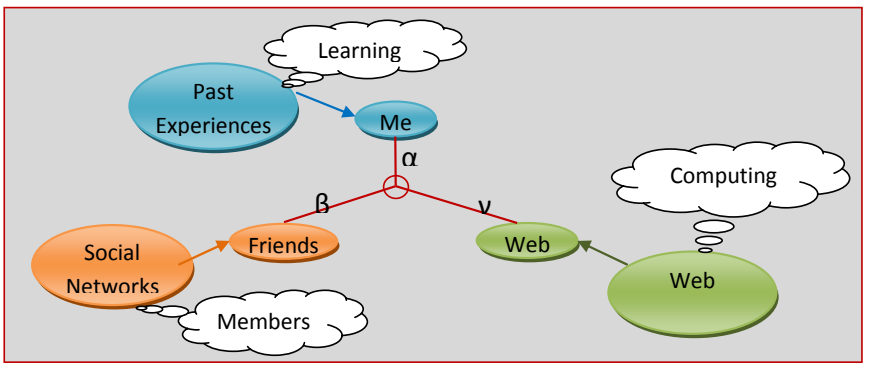

Fig. 1. MFW Model.

The goal of MFW model is to provide optimal (personalized) navigation assistance using navigation quality of services (NavQoS). One of the objectives of MFW is that it should provide navigation assistance to people and in situations where existing navigation systems/services are not able to.

MFW is based on an equilibrium triangle as shown in Fig. 1 where nodes represent "Me", "Friends", and "Web" and links represent the relationships among them. Each link in this triangle has a "degree of influence": $\alpha$ (degree of influence by "Me"), $\beta$ (degree of influence by "Friends"), and $\gamma$ (degree of influence by "Web"), indicating the degree with which an entity influences the focused final decisions and an associated weight $(w)$ indicating the importance of the information provided by an entity ("Me", "Friends", "Web"). Each entity, depending on the roles and the situation at hand, may be assigned a different "degree of influence" and a different weight. The weights are used to calculate the "confidence factor" (C) as follows:

$$
C=\operatorname{Max}\left(\frac{\sum_{i=1}^{n} \alpha_{i}}{n} w_{\alpha}, \frac{\sum_{j=1}^{m} \beta_{j}}{m} w_{\beta}, \frac{\sum_{k=1}^{p} \gamma_{k}}{p} w_{\gamma}\right)
$$

with $w_{\alpha}+w_{\beta}+w_{\delta}=1$.

$$
\begin{array}{ll}
C \prec \delta & \text { Recommend with low confidence } \\
C \geq \delta & \text { Recommend with high confidence }
\end{array}
$$

where $\alpha, \beta, \gamma$ are degrees of influence by "Me", "Friends", and "Web", respectively, $\mathrm{n}, \mathrm{m}, \mathrm{p}$ are number of possible options by "Me", number of social referrals by "Friends" sharing their experiences, and number of Web resources in "Web" with similar computations, respectively, ${ }^{w}$ is weight indicating priority of each entity, and $\delta$ is a threshold that determines the degree to which the provided information is of value to the user.

Note that the degree of influence in MWF is different that the degree of influence suggested by others. For example, [8] defines the degree of influence (I) in any social network as a function of the number of members in the network, the strength of each member, and the distance between the members, as expressed as follows:

$$
I_{i}=\left(\sum_{1}^{N}\left(\frac{s_{j}}{d_{i j}}\right)^{2}\right)^{1 / 2}
$$

where $s_{j}$ is each member's strength and $d_{i j}$ is the distance between members $i$ and $j$.

MFW makes two important recommendations, one on POIs and one on routes. Provided below are explanations of how MFW makes POI recommendations and route recommendations.

\section{A. POI Recommendation}

MFW considers three recommendation strengths on POIs: Low, Medium, or High. Users request POIs by sending a request to SoNavNet with the assumption that they prefer to receive information from trusted members within SoNavNet. MFW tries to find an optimal solution by combining knowledge of the user, trusted members recommendations, and Web resources. We define "POI referral" as number of POI exchanges between each pair of members. This referral has a direction, as user A might refer to user B many times, but user B may refer to user C rather than user A for POI recommendation. Thus, in SoNavNet, the direction of information flow between each set of nodes (members) denotes the POI referral between members. Let $N R_{i j}$ denote the number of POI referrals by individual $i$ to individual $j$. Initially, it can be assumed that POI referrals are equal for all members, i.e., $N R_{i j}=N R_{j i}=1$, but over time $N R$, in one direction or both directions, will increase as more referrals are made.

MFW tries to find a POI referral (member) within SoNavNet whose recommendation meets user's requested NavQoS. The probability of POI referral of $i$ to $j, P_{i j}$, is defined as follows:

$$
P_{i j}=\frac{N R_{i j}}{N R_{i j}+N R_{j i}}
$$

with the condition that $P_{i j}+P_{j i}=1$. Thus, in the initial stage when there are no POI referrals in SoNavNet, $N R_{i j}=N R_{i j}=1$ and $P_{i j}=P_{j i}=0.5$.

Difference between values of $P_{i j}$ and $P_{j i}$ indicates how much $i$ and $j$ refer to each other to exchange POIs. The less the difference, the more the chances of referrals, unless $N R=$ 1 , which means that these members have never referred to each other for POI exchange. Once user $i$ refers to user $j$, the value of $N R_{i j}$ increments by one.

The same concept can be applied to the relationship between social networks. That is, social network $A$ is connected to social network $B$ through a weight, $N R$. Initially, $N R$ 's value is 1 . However, the value of $N R$ will increase as more navigation referrals between social networks are made. The idea behind having connections between social networks 
is that if SoNavNet through MFW cannot find a navigation solution or a reliable solution for a request, it can refer to another social network to find a reliable solution. In MFW, NavQoS is defined as follows:

$$
\text { NavQoS }=T * R
$$

where $T$ and $R$ represent the distinct values for Type and Recommendation, respectively.

Social matching (SM) is defined as follows:

$$
S M=\frac{P_{i A}}{\left(T_{i}-T_{A}\right) *\left(R_{i}-R_{A}\right)}
$$

where $i$ corresponds to a member of SoNavNet whose recommendation is considered and A corresponds to the requester.

The more the value of $P_{i A}$ (i.e., close to 0.5 ) and the less the value of $\left(T_{i}-T_{A}\right) *\left(R_{i}-R_{A}\right)$ (i.e., close to zero), the higher the social match, i.e., the recommended POI socially matches user's request.

\section{B. Route Recommendation}

For recommendation on a route, MFW considers several criteria such as Shortest Distance (SD), Fastest Time (FT), Least Turns (LT), and Least Elevation (LE) change. Each criterion can be assigned a value between 0 and 1 . However, the sum of the values must be equal to 1 . Members who recommend routes are encouraged to initialize the recommended route's criterion and the level of each recommendation, R, i.e., Low, Medium, or High. If users do not enter route criteria, those specified in their profiles are used.

There could be two cases when user A requests a route from Origin $(\mathrm{O})$ to Destination (D), with for example SD = $0.5, \mathrm{FT}=0.3, \mathrm{LT}=0.2$. In the first case, MFW searches for a route from $\mathrm{O}$ to $\mathrm{D}$ with criteria equal or close to values 0.5 , 0.2 , and 0.2 , respectively. In the second case, MFW is not able to find a route from $\mathrm{O}$ to $\mathrm{D}$, but it finds one or more routes that overlap candidate routes between $\mathrm{O}$ and $\mathrm{D}$ and have equal or close criteria values. If these routes pass through O-D, the one with closest match is returned to the user.

In both cases, either finding a path from $\mathrm{O}$ to $\mathrm{D}$ or a path that passes through $\mathrm{O}$ and $\mathrm{D}$, NavQoS is defined as follows:

$$
\mathrm{NavQoS}=\mathrm{ST} * \mathrm{FT} * \mathrm{LT}^{*} \mathrm{R}
$$

where the set of $\{1,2,3\}$ corresponds to the values for $\mathrm{R}$, i.e., \{Low, Medium, High\}.

In order to select an appropriate route recommendation among the available ones, matching score, which is defined as follows, must be greater than or equal a threshold.

$$
S M=\frac{P_{A I}}{\left(S T_{i}-S T_{A}\right) *\left(F T_{i}-S T_{A}\right) *\left(L T_{i}-L T_{A}\right) *\left(R_{i}-R_{A}\right)}(7)
$$

where $i$ corresponds to the recommendation from member $I, A$ corresponds to the requester, and $P_{A i}$ is the value of "route referral" between $A$ and $i$.

The more the value of $P_{A i}$ (i.e., close to 0.5), and the lower the value of $\left(S T_{i}-S T_{A}\right) *\left(F T_{i}-F T_{A}\right) *\left(L T_{i}-L T_{A}\right) *\left(R_{i}-R_{A}\right)$ (i.e., close to zero), the higher the social matching score, i.e., the more the recommended route socially matches user's request.

\section{NAVIGATION SySTEMS/SERVICES AND SoNAVNET/MFW}

To better understand navigation assistance that can be provided by navigation systems/services and the SoNavNet/MFW approach, Table 1 highlights the key differences between these two approaches. In this table, computed and recommended POIs, routes, and directions by these two approaches are compared and analyzed.

Upon a POI request, navigation systems/services search their databases to find a match POI, which could be in outdoors or indoors. Navigation systems/services typically contain published POIs (such as information published in yellow pages) with general information on POIs. The output of a POI request is either the matched POI if the system/service can find an exact match or empty if there is no exact match in the database. The main algorithm finds an exact match in the database for the requested POI. SoNavNet/MFW searches its database of all recommended POIs and WMSs to find a match POI, which could be in outdoors or indoors. SoNavNet/MFW's POI database contains POIs that are of members interest with detailed annotation on POIs. The output of a POI request is either the matched POI if SoNavNet/MFW can find a close match in the database or WMSs or empty if there is no close match in the database or WMSs. The main algorithm tries to find a close match in the database or WMSs for the requested POI.

Upon a route request, navigation systems/services compute a route using networks, roads and sidewalks in outdoors and hallways in indoors. Most current navigation systems/services can compute routes by taking a single criterion (e.g., shortest distance) into account; routes computed based on a singlecriterion are considered general-purpose routes. The output is a route and the main algorithm is an optimization algorithm. SoNavNet/MFW searches the database of all recommended routes and WMSs to find a close match in outdoors, using roads and sidewalks, and indoors, using hallways. SoNavNet/MFW's route database contains routes that are of members interest which implicitly take into account multiple combined criteria; routes computed based on multiple-criteria are considered personalized routes. The output of a requested route is either the matched route if SoNavNet/MFW can find a close match in its database or WMSs or empty if there is no close match in the database or WMSs. The main algorithm finds a close match in the database or WMSs for the requested route. 
TABLE 1

DIFFERENCES BETWEEN NAVIGATION SYSTEMS/SERVICES AND SONAVTNET/MFW

\begin{tabular}{|c|c|c|c|}
\hline Navigation Assistance & POI & Route & Direction \\
\hline $\begin{array}{c}\text { Navigation } \\
\text { Systems/Services }\end{array}$ & $\begin{array}{c}\text { Search database of available POIs } \\
\text { Outdoor: POIs in outdoors (e.g., } \\
\text { restaurant) } \\
\text { Indoor: POIs in buildings (e.g., } \\
\text { office) } \\
\text { Published information } \\
\text { General information } \\
\text { Output: POI (if in database) or null } \\
\text { (if not in database) } \\
\text { Algorithm: data retrieval }\end{array}$ & $\begin{array}{l}\text { Compute using networks } \\
\text { Outdoor: roads/sidewalks } \\
\text { Indoor: hallways } \\
\text { Single criterion } \\
\text { General route } \\
\text { Output: route } \\
\text { Algorithm: optimization }\end{array}$ & $\begin{array}{c}\text { Compute using networks } \\
\text { Outdoor: roads/sidewalks and } \\
\text { attributes in database } \\
\text { Indoor: hallways and attributes in } \\
\text { database } \\
\text { Limited criteria } \\
\text { General characteristics/landmarks } \\
\text { Output: direction } \\
\text { Algorithm: geometrical and } \\
\text { topological computations and data } \\
\text { retrieval }\end{array}$ \\
\hline SoNavNet/MFW & $\begin{array}{c}\text { Search database of recommended } \\
\text { POIs } \\
\text { Outdoor: POIs in outdoors (e.g., } \\
\text { gas station) } \\
\text { Indoor: POIs in indoors (e.g., water } \\
\text { fountain) } \\
\text { Detailed annotation } \\
\text { Personalized information } \\
\text { Output: POI (if in database) or null } \\
\text { (if not in database) } \\
\text { Algorithm: matching }\end{array}$ & $\begin{array}{c}\text { Search database of recommended } \\
\text { routes } \\
\text { Outdoor: roads/sidewalks } \\
\text { Indoor: hallways } \\
\text { Multiple criteria } \\
\text { Personalized route } \\
\text { Output: route (if in database) or null } \\
\text { (if not in database) } \\
\text { Algorithm: matching }\end{array}$ & $\begin{array}{c}\text { Search database of recommended } \\
\text { directions } \\
\text { Outdoor: roads/sidewalks } \\
\text { Indoor: hallways } \\
\text { Multiple criteria } \\
\text { Personalized } \\
\text { characteristics/landmarks } \\
\text { Output: direction } \\
\text { Algorithm: matching }\end{array}$ \\
\hline
\end{tabular}

Upon a direction request, navigation systems/services compute a set of instructions using networks, roads and sidewalks along with attributes in database in outdoors and hallways along with attributes in database, in indoors. Most current navigation systems/services compute directions by taking into account a limited set of criteria into account, directions computed based on limited criteria are considered general-purpose directions. The output is a direction and the main algorithm performs geometrical and topological computations and retrieves data from database. SoNavNet/MFW searches the database of all directions recommended by its members and WMSs to find a close match in outdoors, using roads and sidewalks along with attributes in database, and indoors, using hallways along with attributes in database. SoNavNet/MFW's direction database contains directions that are of members interest which can take into account multiple combined criteria; directions computed based on multiple-criteria are considered personalized directions. The output is a direction and the main algorithm finds a close match in the database or WMs for the requested direction.

Fig. 2 highlights navigation assistance, provided by navigation systems/services and SoNavNet/MFW, that can be for the general population, for specific groups (e.g., wheelchair users), or for individuals. As shown in the figure, level of acceptance of navigation assistance could be low to high and that SoNavNet/MFW is able to provide a higher level of acceptance than navigation systems/services, especially at individual level.

Fig. 3 shows database growth, containing mainly POIs, in navigation systems/services and SoNavNet/MFW. Initially, navigation systems/services start with a large database of POIs but over time, the size of the database grows as new POIs are added to the database. SoNavNet/MFW, on the other hand, may start with no POIs in its database, but as members use the network and recommend new POIs, the database size is expected to grow tremendously in a short time.

Fig. 4 shows the relationship between route/direction options in navigation systems/services and SoNavNet/MFW. As shown in the figure, while current navigation systems/services provide routes/directions mostly addressing the needs and preferences of the general population, there can provide route choices closer to the needs and preferences of groups. SoNavNet/MFW, on the other hand, is expected to provide routes/directions that closely meet the needs and preferences of individuals as the network is used by members and new and different routes/directions are recommended.

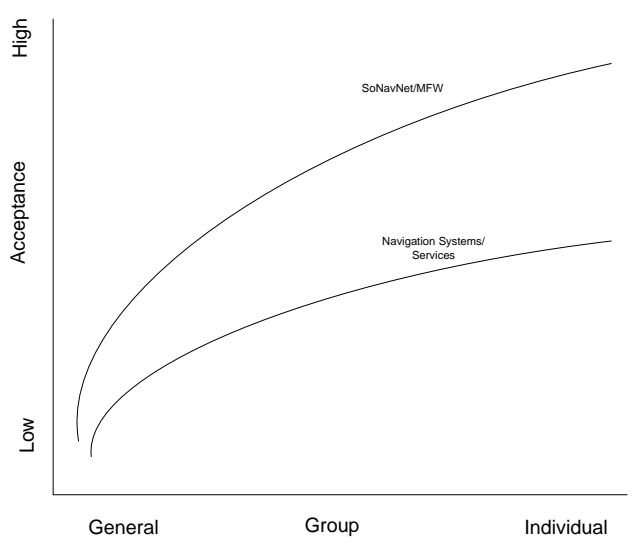

Fig. 2. Acceptance of navigation assistance by navigation systems/services and SoNavNet/MFW. 


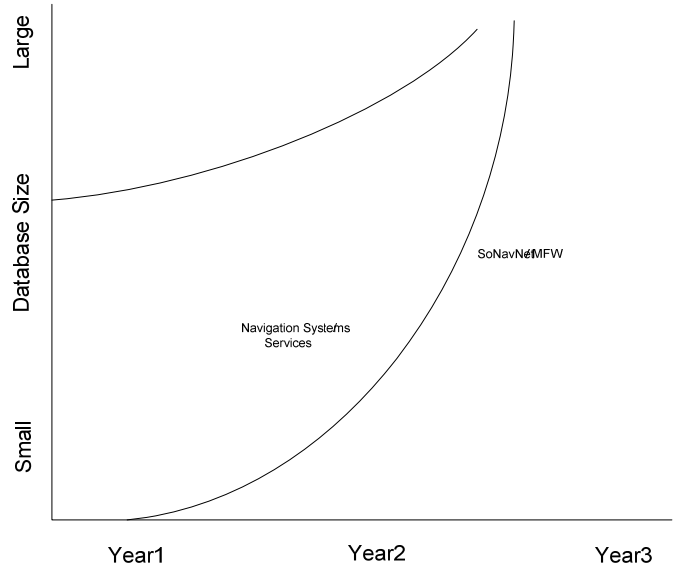

Fig. 3. Growth of databases (POIs) in navigation systems/services and SoNavNet/MFW.

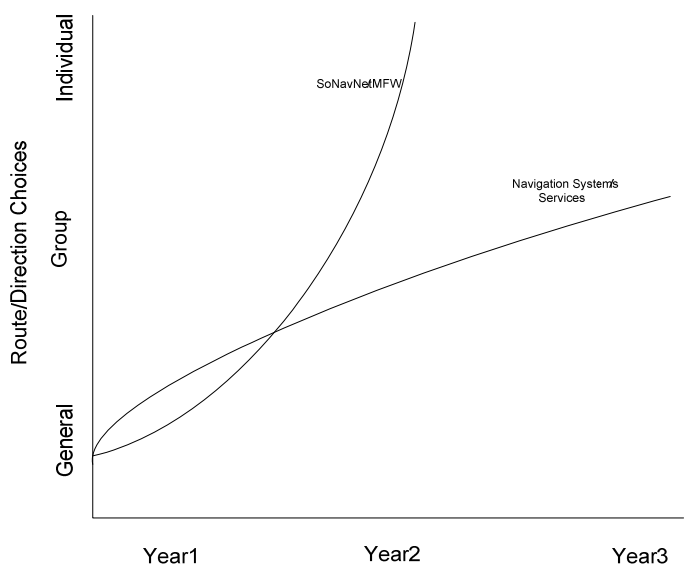

Fig. 4. Route choices by navigation systems/services and SoNavNet/MFW.

\section{SUMMARY AND FUTURE RESEARCH}

Navigation systems/services have become commonplace and people increasingly rely on them for navigation assistance. While modern navigation systems/services have become computationally sophisticated gadgets that can provide general-purpose navigation assistance, they fall short in addressing the navigation needs and preferences at the individual level, among other things. To overcome the shortcomings of current navigation systems/services, SoNavNet has been developed. SoNavNet, unlike navigation systems/services that base their solutions on computational models and algorithms, provides navigation assistance through experiences of people and available information on the Web. This means that SoNavNet can address the navigation needs and preferences at the individual level without the need for development of complex mathematical models and algorithms. MFW in SoNavNet, as a social navigation network system, can facilitate personalization of navigation assistance by considering all available user's knowledge and experiences, recommendations by user's friends, and information and computations available through WMSs, among other Web resources. In short, MFW in SoNavNet can provide personalized navigation assistance anywhere and anytime.

In our future research, once MFW is fully developed and incorporated into SoNavNet, we will:

- Develop appropriate algorithms and information filtering to address the problem of combining multiple sources to provide optimal navigation assistance.

- Demonstrate the benefits of the MFW model through a specific proof of concept with some of the MFW application's features and capabilities.

- Evaluate MFW as a guide to building future collaborative systems for location-based recommendations. The ability to provide navigation assistance that provides information based on multiple constraints will extend existing work on recommendation systems, route-finding algorithms, and information extraction.

\section{REFERENCES}

[1] H. A. Karimi, B. Zimmerman, A. Ozcelik, and D. Roongpiboonsopit, (2009a) "SoNavNet: A framework for social navigation networks," in Conf. on Location Based Social Networks, Seattle, Nov 3, 2009.

[2] K. O'Keefe, (2001) “Availability and reliability advantages of GPS/Galileo integration,”. in Proc. of ION GPS, Salt Lake City, UT, Sep. 11- 14, pp.2096-2104, 2001.

[3] W. Y. Ochieng, K. Sauer, P. A. Cross, K. F. Sheridan, J. Iliffe, S. Lannelongue, N. Ammour, and K. Petit, "Potential performance levels of a combined Galileo/GPS navigation system,".J of Navigation, vol. 54, no. 2, pp. 185-197, 2001.

[4] H. A. Karimi, D. Nwan, and B. Zimmerman, "Navigation assistance through "Models" or "Experiences”?," GIM International, December, 2009.

[5] P. Kasemsuppakorn and H. A. Karimi, "Pedestrian network data collection through location-based social networks," in $5^{\text {th }}$ Int. Conf. on Collaborative Computing: Networking, Applications and Worksharing, Washington, DC, Nov. 11-14, 2009.

[6] M. Anwar, H. A. Karimi, and J. G. Benner, "OnLocEd: Finding learning resources and communities," in $3^{\text {rd }}$ Int. Conf. on Computer Supported Education, Noordwijkerhout, the Netherlands, May 6-9, 2011.

[7] H. A. Karimi, J. G. Benner, and M. Anwar, "A model for navigation experience sharing through social navigation networks (SoNavNets)," in Int. Workshop on Issues and Challenges in Social Computing (WICSOC2011), Las Vegas, August 2, 2011.

[8] A. Nowak and R. Vallacher. "Information and Influence in the Construction of Shared Reality”, IEEE Intelligent System, pp. 90-93, 2005 